\title{
Proton Spin Relaxation in Solutions of the Complex Tris(acetylacetonato)chromium(III)
}

\author{
Pei-Lein WANG, ${ }^{*}$ Jing-HUEI LeE, ${ }^{*}$ Shiou-Mei HuANG, ${ }^{*}$ \\ AND LIAN-PIN HWANG* $+\ddagger$ \\ * Department of Chemistry, National Taiwan University, Taipei, Taiwan, Republic of China, and \\ $\dagger$ Institute of Atomic and Molecular Science, Institute of Biological Chemistry, \\ Academia Sinica, Nankang, Taipei. Taiwan, Republic of China
}

Received October 24, 1986; revised December 31, 1986

\begin{abstract}
Field-dependent proton spin-lattice and spin-spin relaxation times have been measured for $\mathrm{CH}_{3}$ in dilute chloroform solutions of tris(acetylacetonato)chromium(III) (Cr(acac) complex over a temperature range of 253-323 $\mathrm{K}$ and a proton resonance frequency range of $10-90 \mathrm{MHz}$. The results have been interpreted in terms of scalar, dipolar, and scalardipolar cross relaxation. The effects of methyl group internal rotation are included. In particular, coupled modulation of ESR relaxation and electron-nuclear dipolar relaxation by reorientational motion of the complex is treated. The reorientational correlation times of the complex obtained are consistent with those measured from ${ }^{13} \mathrm{C} T_{1}$ in the analogous diamagnetic Co(III) complex, but significant deviations from the Stokes-Einstein relation with the consideration of microviscosity are observed. Solvation of the complex with chloroform is further identified from chemical-shift measurements and is utilized to interpret the results. The proton $T_{1}$ data are discussed in terms of their relevance to the zero-fieldsplitting interaction and to the contribution of scalar-dipolar cross relaxation. (6) 1987 Academic Press, Inc.
\end{abstract}

\section{INTRODUCTION}

For most transition metal complexes with $S \geqslant 1$, the electron relaxation processes in solution are attributed to the fluctuation of the zero-field-splitting (ZFS) interaction (1). The transition metal complex tris(acetylacetonato)chromium(III) $\left(S=\frac{3}{2}\right)$ has been used as an inert, organic-soluble relaxation enhancing reagent in solution (2). However, the detailed spin relaxation processes of this complex in solution are not known completely. An understanding of spin relaxation processes may be needed for investigating the quantitative effects on NMR relaxation. Doddrell et al. (3) studied proton relaxation of this complex in $\mathrm{CDCl}_{3}$ solutions. They assumed that the methyl group jumps among positions of a three-minimum potential. Independent modulations of dipolar and ZFS interactions by the same reorientational motion were assumed. Second-order perturbation theory was used in the treatment of ESR relaxation. Hwang and Ju (4) extended the approach of solving the stochastic Liouville equation to the cases where the nuclear spin is rapidly relaxing due to fast electron relaxation processes for the system with $S$

$\ddagger$ To whom correspondence should be addressed. 
$=\frac{3}{2}$. It is found that the effects of higher-order perturbation of ZFS interaction should be considered for this complex in the slow motional region at low fields. A comparison between coupled and independent modulations of ZFS and dipolar interactions was made. The effects of internal rotation of methyl groups were also examined.

It is the purpose of this work to investigate the role of paramagnetic spin in proton NMR relaxation in the system of dilute $\mathrm{Cr}(\mathrm{acac})_{3}$ solution at various temperatures and magnetic fields. The reorientational correlation times obtained from $T_{1}$ measurements of ${ }^{13} \mathrm{C}$ in $\mathrm{Co}(\mathrm{acac})_{3}$ systems have been considered in the interpretation of proton relaxation data in the $\mathrm{Cr}(\mathrm{acac})_{3}$ system. Free internal rotation of methyl groups in the complex and coupled modulation of ZFS interaction and dipolar interaction between electron-nuclear spins are used in the interpretation of experiments. Solvation of the complex with chloroform is also examined.

\section{EXPERIMENTAL}

Samples. Co(acac) $)_{3}$ was obtained from TCI, Tokyo Kasei, Japan, and used without further purification. $\mathrm{Cr}(\mathrm{acac})_{3}$ was prepared according to the method used in (5). The appropriate amount of $\mathrm{Cr}(\mathrm{acac})_{3}$ was dissolved in $\mathrm{CDCl}_{3}(99.8 \%, \mathrm{E}$. Merck) to make a $0.05 M$ solution. The solutions of $\mathrm{Co}(\mathrm{acac})_{3}$ were degassed with the freeze-pumpthaw cycle technique to eliminate oxygen and then sealed in $10 \mathrm{~mm}$ tubes.

NMR measurements. The ${ }^{13} \mathrm{C}$ and ${ }^{1} \mathrm{H}$ NMR spectra were obtained on a Bruker MSL-90 spectrometer. The spectrometer has a field-frequency lock at $90 \mathrm{MHz}$ only. The measurements were made at $10,20,27,35,45,55,65,80$, and $90 \mathrm{MHz}$, respectively. The $\mathrm{C}-\mathrm{H}$ signal is not apparent in the proton spectrum. The spin-lattice relaxation times were obtained by the inversion-recovery method. The probe temperatures were varied by $10^{\circ}$ intervals from 253 to $323 \mathrm{~K}$. The accuracy in temperature was within $0.5^{\circ} \mathrm{C}$. In the measurement of $T_{1}, 14$ different pulse intervals were used and the delay time was kept longer than five times $T_{1} . T_{2}$ was determined from linewidth measurements. For measurements of $T_{2}$ at 10 and $45 \mathrm{MHz}$, field homogeneity was carefully adjusted with the proton FID signal in $0.01 \mathrm{MCuSO}_{4}$ aqueous solution. Reproducibility of relaxation rates was considerably better than $\pm 10 \%$.

\section{THEORY}

Details of the theory for the interpretation of proton relaxation in paramagnetic systems with $S=\frac{3}{2}$ were recently given by Hwang and $\mathrm{Ju}$ (4). Here, we briefly summarize the formulation of the theory. In the calculation of relaxation rates of nuclear spin, denoted by $I$, due to the presence of electron spin, denoted by $S$, in a paramagnetic complex, it is assumed that the I-S interactions are both dipolar and scalar. The dipolar interaction is assumed to be modulated by the reorientation of the $\mathbf{r}_{\text {IS }}$ vector due to overall Brownian reorientation of the complex and interference from internal rotation of the methyl group. It is also modulated by the electron relaxation processes, while the scalar interaction is modulated by the latter process only. It is usually believed that the reorientational motion of the complex gives rise to modulation of the ZFS interaction. The electron relaxation processes are attributed to the fluctuation of the ZFS interaction. The proton longitudinal relaxation rates in the present case are given 
by

$$
\frac{1}{T_{1}}=\frac{1}{T_{1}^{\mathrm{sc}}}+\frac{1}{T_{1}^{\mathrm{dd}}}+\frac{1}{T_{1}^{\mathrm{sd}}}
$$

where the superscripts indicate scalar, dipolar, and scalar-dipolar cross relaxations, respectively. An analogous expression also holds for the proton spin-spin relaxation rate.

As in the similar but somewhat simpler case treated by Abragam (6), the proton relaxation rates due to scalar interaction are given by

$$
\frac{1}{T_{1}^{\mathrm{sc}}}=A^{2}\left[J_{+1}\left(-\omega_{1}\right)+J_{-1}\left(\omega_{1}\right)\right]
$$

and

$$
\frac{1}{T_{2}^{\mathrm{sc}}}=A^{2}\left[J_{0}(0)+\frac{1}{2} J_{+1}\left(-\omega_{1}\right)+\frac{1}{2} J_{-1}\left(\omega_{\mathrm{I}}\right)\right],
$$

where $A$ is the scalar coupling constant between electron and nuclear spins, taken as $A=1.7 \times 10^{6} \mathrm{~s}^{-1}(3)$ in the following calculations. by

The spectral density $J_{m}(\omega)$, at Larmor frequency $\omega$, in our present notation is given

$$
J_{m}(\omega) \equiv 2^{-|m|} \operatorname{Re} \int_{0}^{\infty}\left\langle S_{m}(0)\left(S_{-m}(0)\right)_{t}\right\rangle e^{i \omega t} d t
$$

where the quantity with subscript $t$ denotes

$$
(\mathcal{O}(0))_{t}=e^{i\left(\mathcal{L}_{\mathrm{s}}+\mathcal{L}_{1}\right) t} \mathcal{O}(0) .
$$

Here $\mathcal{L}_{1}$ is the Liouville operator for the lattice system, and $\mathcal{L}_{\mathrm{s}}$ is the Liouville operator corresponding to the spin Hamiltonian of the paramagnetic system, i.e.,

$$
\mathscr{H} \equiv \mathscr{H}_{0}+\mathscr{H}_{\text {ZFS }}
$$

where $\mathscr{H}_{0}$ is the Zeeman energy of the electron spins. Also $\mathscr{H}_{\text {ZFS }}$ is the ZFS interaction energy. It involves only the fluctuations of the direction of the principal components of ZFS interaction but their magnitude does not change upon fluctuation (1). For simplicity, the ZFS of cylindrical symmetry is considered throughout this work. This approximation may be found in the case of $\mathrm{Cr}(\mathrm{acac})_{3}$. The parameter of ZFS gives $\Delta^{2}$ $=\frac{2}{3} D^{2}+E^{2}$ with $D=1.12 \times 10^{11} \mathrm{~s}^{-1}$ and $E=1.51 \times 10^{9} \mathrm{~s}^{-1}(7)$. Thus, the approximation of $D \gg E$ is made in the calculation.

Similarly, the proton relaxation rates due to dipolar interaction are given by

$$
\frac{1}{T_{1}^{\mathrm{dd}}}=\frac{\left(\gamma_{\mathrm{I}} \gamma_{\mathrm{S}} \hbar\right)^{2}}{4}\left[3 J_{0,1}\left(-\omega_{1}\right)+3 J_{0,-1}\left(\omega_{\mathrm{I}}\right)+6 J_{-1,2}\left(-\omega_{1}\right)+6 J_{1,-2}\left(\omega_{1}\right)+J_{1,0}\left(-\omega_{\mathrm{I}}\right)+J_{-1,0}\left(\omega_{\mathrm{I}}\right)\right]
$$

and

$$
\begin{aligned}
\frac{1}{T_{2}^{\mathrm{dd}}=\frac{\left(\gamma_{\mathrm{I}} \gamma_{\mathrm{s}} \hbar\right)^{2}}{4}}\left[\frac{3}{2} J_{0,-1}\left(\omega_{1}\right)\right. & +\frac{3}{2} J_{0,1}\left(-\omega_{1}\right)+\frac{1}{2} J_{-1,0}\left(\omega_{1}\right)+\frac{1}{2} J_{1,0}\left(-\omega_{1}\right) \\
& \left.+3 J_{1,-2}\left(\omega_{1}\right)+3 J_{-1,2}\left(-\omega_{1}\right)+3 J_{-1,-1}(0)+3 J_{1,1}(0)+4 J_{0,0}(0)\right]
\end{aligned}
$$


where $J_{m, n}(\omega)$ is the spectral density at the Larmor precession frequency $\omega$, as defined by

$$
J_{m, n}(\omega) \equiv 2^{2-|m|} \operatorname{Re} \int_{0}^{\infty} e^{i \omega t}\left\langle D_{0 n}^{2}(\Omega) S_{m}(0) / r_{1 \mathrm{~S}}^{3} \times\left(D_{0 n}^{2}(\Omega) S_{m}(0) / r_{1 \mathrm{~S}}^{3}\right)_{i}^{*}\right\rangle d t,
$$

where $D_{m n}^{\mathrm{L}}(\Omega)$ are the elements of the Wigner rotation matrix $(8)$ and the Euler angle $\Omega$ specifies the orientation of $\mathbf{r}_{i S}$ in the laboratory frame. Here, for the so-called coupledrotation model (CRM), the time-correlation function is evaluated with the coupling of spin and orientation variables.

The cross relaxation rates due to scalar and dipolar interactions may be expressed by

$$
\frac{1}{T_{1}^{\mathrm{s}}}=\frac{\hbar}{2} \gamma_{\mathrm{I}} \gamma_{\mathrm{S}} A\left[K_{-1}\left(\omega_{1}\right)+K_{+1}\left(-\omega_{\mathrm{I}}\right)+L_{-1}\left(\omega_{\mathrm{I}}\right)+L_{+1}\left(-\omega_{1}\right)\right]
$$

and

$$
\frac{1}{T_{2}^{\mathrm{sd}}}=\gamma_{1} \gamma_{\mathrm{S}} A \hbar\left\{-4\left[L_{0}(0)+K_{0}(0)\right]+\frac{1}{2}\left[L_{+1}\left(-\omega_{\mathrm{I}}\right)+K_{+1}\left(-\omega_{\mathrm{I}}\right)\right]+\frac{1}{2}\left[L_{-1}\left(\omega_{\mathrm{I}}\right)+K_{-1}\left(\omega_{\mathrm{I}}\right)\right]\right\},
$$

where the spectral density is defined by

$$
K_{m}(\omega)=\operatorname{Re} \int_{0}^{\infty} d t e^{j \omega t}\left\langle S_{m}(0) D_{00}^{2}(\Omega) r_{\mathrm{IS}}^{-3}\left(S_{-m}(0)\right)_{t}\right\rangle
$$

and

$$
L_{m}(\omega)=\operatorname{Re} \int_{0}^{\infty} d t e^{j \omega t}\left\langle S_{m}(0)\left(S_{-m}(0) D_{00}^{2}(\Omega) r_{1 \mathrm{IS}}^{-3}\right)_{l}\right\rangle
$$

For methyl proton relaxation in $\mathrm{Cr}(\mathrm{acac})_{3}$ solution, the internal rotation of the methyl group not only changes the orientation of $r_{\text {Is }}$ but also changes its magnitude. Here, we assumed that only the motions for internal rotation of methyl groups and for overall reorientation of the complex need be considered. They are treated independently. If isotropic reorientational diffusion of the complex is still assumed, Eqs. [9] and [12] may be simplified as

$$
J_{m, n}(\omega)=2^{2-|m|} \operatorname{Re} \int_{0}^{\infty} d t e^{j \omega t} \sum_{q} \Gamma_{q q}\left\langle D_{q n}^{2}(\Omega) S_{m}(0)\left(D_{q m}^{2}(\Omega) S_{m}(0)\right)_{i}^{*}\right\rangle
$$

and

$$
K_{m}(\omega)=\operatorname{Re} \int_{0}^{\infty} d t e^{i \omega t} \sum_{q} \Gamma_{q}\left\langle S_{m}(0) D_{q 0}^{2}(\Omega)\left(S_{-\mathrm{m}}(0)\right)_{t}\right\rangle
$$

Analogous expressions may be written for $L_{m}(\omega)$ as well.

In Eqs. [14] and [15] we have defined

and

$$
\Gamma_{q q}=\left\langle\left(D_{0 q}^{2}\left(\Omega^{\prime}\right) / r_{\mathrm{IS}}^{3}\right)\left(D_{0 q}^{* 2}\left(\Omega^{\prime}\right) / r_{\mathrm{IS}}^{3}\right)_{t}\right\rangle^{\prime}
$$

$$
\Gamma_{q}=\left\langle D_{0 q}^{2}\left(\Omega^{\prime}\right) / r_{1 \mathrm{~S}}^{3}\right\rangle^{\prime},
$$


where $\langle>$ 'denotes the equilibrium average over internal rotation around the $\mathrm{C} 3$ axis of the methyl group, and $\Omega^{\prime}$ is defined as the orientation of vector $\mathbf{r}_{I S}$ with respect to the frame specified by the direction of the $\mathrm{C} 3$ axis. Also, the orientation $\Omega$ is defined as the orientation of the $\mathrm{C} 3$ frame with respect to the laboratory axis. For simplicity, it is assumed that the principal axis coincides with the direction of the $\mathrm{C} 3$ axis. It should be noted that Eq. [16] is part of the integrand in the evaluation of dipolar spectral density (cf. Eq. [9]). Furthermore, we treat the internal rotation as an isotropic Brownian diffusion. The time-correlation function for internal rotation can be evaluated with the help of bond lengths and angles obtained from crystal data (9). This yields

$$
\begin{aligned}
\sum_{q} \Gamma_{q q}=\left(8.13638 \times 10^{43}+\right. & 1.42436 \times 10^{43} e^{-R^{\prime} t}+4.24746 \times 10^{41} e^{-4 R^{\prime} t} \\
+ & \left.8.19735 \times 10^{39} e^{-9 R^{\prime} t}+1.10168 \times 10^{38} e^{-16 R^{\prime} t}\right) \mathrm{cm}^{-6},
\end{aligned}
$$

where $R^{\prime}$ is the diffusion coefficient for internal rotation. It is found that internal barriers of the methyl group in carbonyl systems are less than $1 \mathrm{kcal} / \mathrm{mole}(10)$. When the free rotation of the methyl group is considered in this work, we have

$$
R_{0}^{\prime}=(k T / I)^{1 / 2}=0.86 \times 10^{13} \mathrm{~s}^{-1} \quad\left(20^{\circ} \mathrm{C}\right),
$$

where $I$ is the moment of inertia of the methyl group.

To get the results described in the next section, the spectral density functions Eqs. [4], [9], [12], and [13] were evaluated numerically for the overall reorientation of the complex by the approach of solving the stochastic Liouville equation. This method has been developed as a general approach to spin relaxation processes $(11)$. The detailed numerical method is discussed elsewhere.

\section{RESULTS AND DISCUSSIONS}

Since the $\mathrm{Cr}^{+3}$ ion is similar in size to the $\mathrm{Co}^{+3}$ ion, the overall reorientational correlation times, determined from ${ }^{13} \mathrm{C}$ relaxation, of the $\mathrm{Co}(\mathrm{acac})_{3}$ complex in solutions are adopted for $\operatorname{Cr}(\mathrm{acac})_{3}$. The observed spin-lattice relaxation times for methyl and methine carbons in $\mathrm{Co}(\mathrm{acac})_{3}$ are listed in Table 1 . It is believed that dipolar interaction between $\mathrm{C}-\mathrm{H}$, modulated by the reorientational motion, is the dominant mechanism for methine ${ }^{13} \mathrm{C}$ relaxation in $\mathrm{Co}(\mathrm{acac})_{3}$. Furthermore, due to low internal rotation barriers of the methyl group, free internal rotation of the methyl group (cf. Eq. [19]) may be assumed. Thus, the overall reorientational correlation times may be determined in accordance with Woessner's equation with the help of methyl ${ }^{13} \mathrm{C} T_{1}$ data $(10)$. Using $r_{\mathrm{C}-\mathrm{H}}=1.10 \AA$ (12), the reorientational correlation times calculated from ${ }^{13} \mathrm{C}-$ $\mathrm{H}$ data are obtained and also listed in the table. It is found that the agreement between two reorientational correlation times obtained from methyl and methine ${ }^{13} \mathrm{C}$ data, respectively, is satisfactory.

As studied by Hwang and $\mathrm{Ju}$, it was found that the effect of ESR relaxation on proton $T_{1}$ in $\mathrm{Cr}(\mathrm{acac})_{3}$ appears only at low field. Field-dependent studies of relaxation rates may be useful in revealing this effect. Figure 1 shows the frequency dependence of the observed proton $1 / T_{1}$ for various temperatures cited. The calculated relaxation rates are also shown for comparison. The reorientational correlation times used in the 


\section{TABLE 1}

Carbon-13 $T_{1}$ Data and Overall Reorientational Correlation Times of $\mathrm{Co}(\mathrm{acac})_{3}$ and $\mathrm{Cr}(\mathrm{acac})_{3}$ in Chloroform Solutions

\begin{tabular}{cccccc}
$\begin{array}{c}\text { Temperature } \\
(\mathrm{K})\end{array}$ & $T_{1}^{\mathrm{CH}}(\mathrm{s})$ & $T_{1}^{\mathrm{CH}_{3}}(\mathrm{~s})$ & $\tau_{\mathrm{CH}}(\mathrm{ps})^{a}$ & $\tau_{\mathrm{CH}_{3}}(\mathrm{ps})^{a}$ & $\tau(\mathrm{ps})^{b}$ \\
\hline 323 & 1.2 & 3.5 & 40 & 39 & 33 \\
313 & 1.1 & 3.2 & 44 & 42 & 43 \\
303 & 1.0 & 2.9 & 48 & 47 & 48 \\
293 & 0.79 & 2.5 & 61 & 55 & 57 \\
283 & 0.65 & 2.0 & 69 & 68 & 74 \\
273 & 0.56 & 1.7 & 86 & 80 & 86 \\
263 & 0.44 & 1.2 & 109 & 114 & 110 \\
253 & 0.35 & 0.95 & 137 & 144 & 138 \\
\hline
\end{tabular}

${ }^{a} \tau_{\mathrm{CH}}$ and $\tau_{\mathrm{CH}_{3}}$ represent the overall reorientational correlation times obtained from $T_{1}$ data of methine and methyl carbon-13, respectively, in $\mathrm{Co}(\mathrm{acac})_{3}$ solutions.

${ }^{b} \tau$ represents the overall reorientational correlation time obtained from proton $T_{1}$ data in $\mathrm{Cr}(\mathrm{acac})_{3}$ solution.

fitting of the experimental data are listed in Table 1. We find that the agreement with those obtained from $\mathrm{Co}(\mathrm{acac})_{3}$ data is excellent. This suggests that the structure of the ligand should be quite static. The proton spin-lattice relaxation rate is almost field-

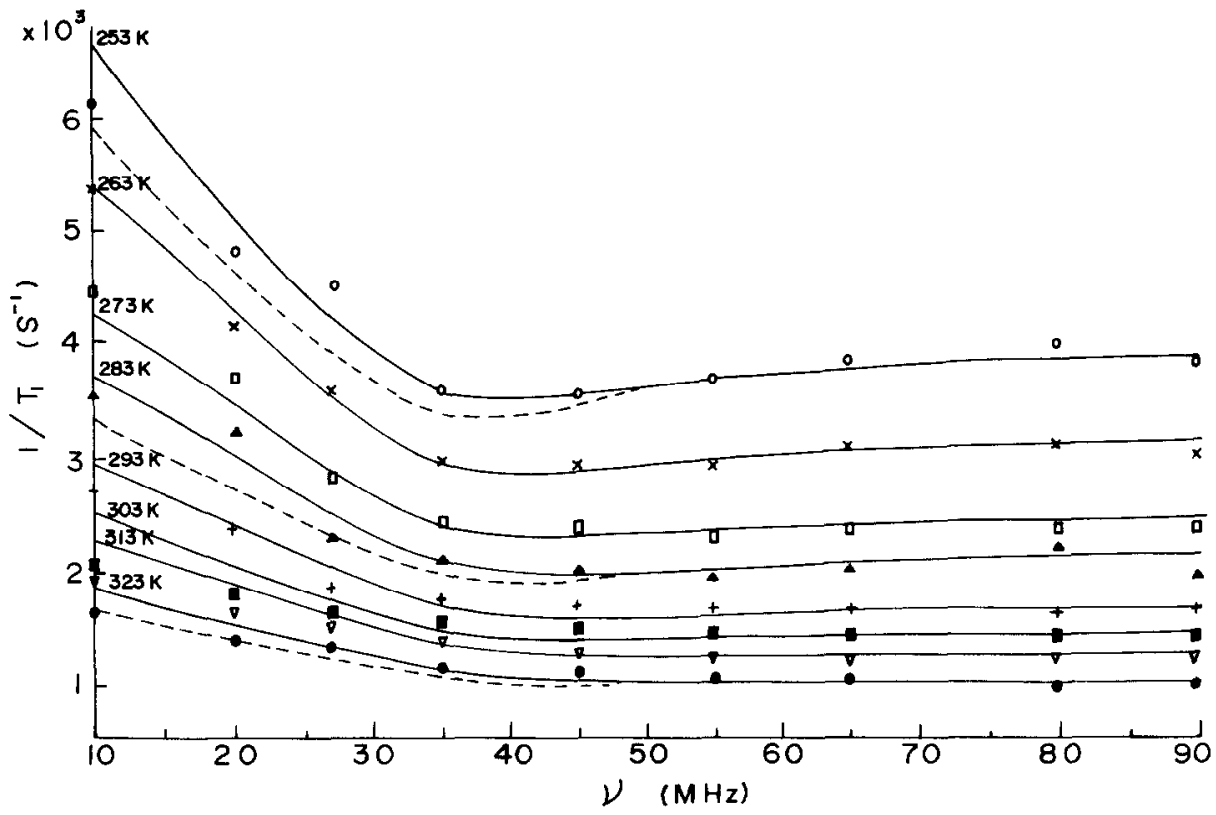

FIG. 1. Proton magnetic relaxation rates in a $0.05 \mathrm{MCr}(\mathrm{acac})_{3}$ chloroform solution as a function of proton resonance frequency for different temperatures cited. The dashed curves refer to the field-dependent $1 / T_{1}^{\text {dd }}$ values. 
independent in the region above $30 \mathrm{MHz}$. This may be explained by the fact that the reorientational modulation of dipolar interaction between $I$ and $S$ is the principal mechanism for proton spin-lattice relaxation and the contribution of ESR relaxation is negligible. It is clear that the ESR relaxation is slow compared to the reorientational motion in this region. Furthermore, the general trends of the observed $1 / T_{1}$ for different temperatures with variation of fields are similar and the differences with temperatures are due to their reorientational rates of the complex. In practice it is found that the contribution of scalar interaction in $T_{1}$ relaxation is only significant at low fields. The calculated relaxation rates with consideration of $1 / T_{1}^{\text {dd }}$ term exclusively at 253,283 , and $323 \mathrm{~K}$ are also shown in the same figure for comparison. Below $30 \mathrm{MHz}$, it is found that the coupling through reorientational motion between dipolar and ESR relaxation becomes important. The calculated $1 / T_{1}$ as a function of ZFS at $10 \mathrm{MHz}$ and $303 \mathrm{~K}$ is shown in Fig. 2. The relaxation rate increases with the magnitude of ZFS for $D<10^{11} \mathrm{~s}^{-1}$, but above this value it varies slightly. Similar behavior is also found for other temperatures.

In Fig. 3, we have shown the comparison of the observed $1 / T_{2}$ with theoretical calculations as a function of temperature at 10,45 , and $90 \mathrm{MHz}$. In the calculations, $D=1.12 \times 10^{11} \mathrm{~s}^{-1}$ and $D=1.30 \times 10^{11} \mathrm{~s}^{-1}$ are used for comparison purposes. For results at $10 \mathrm{MHz}$, the variation of $D$ does not improve the fitting with the experiments. In those calculations, the contribution of the scalar-dipolar cross term in spin-spin relaxation is of importance only at low fields. The neglect of this term gives a $25 \%$ reduction in the relaxation rate at $10 \mathrm{MHz}$ field and about a $10 \%$ reduction at fields above $45 \mathrm{MHz}$.

In the discussion of reorientational diffusion in liquids, one usually interprets the difference between the calculated and the experimental values in terms of microviscosity

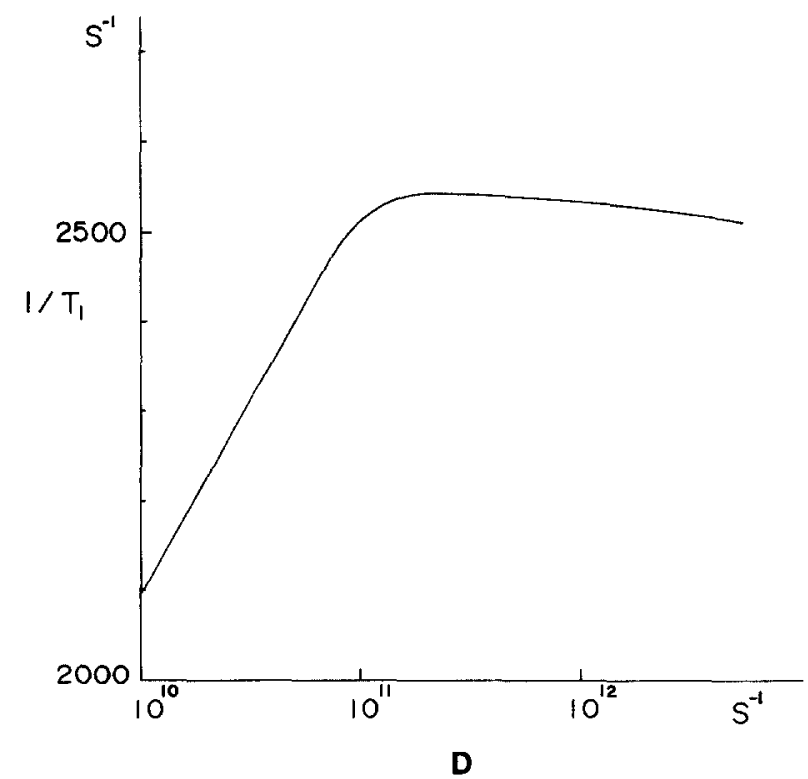

FIG. 2. Calculated proton spin-lattice relaxation rate versus the strength of zero-field-splitting interaction. 


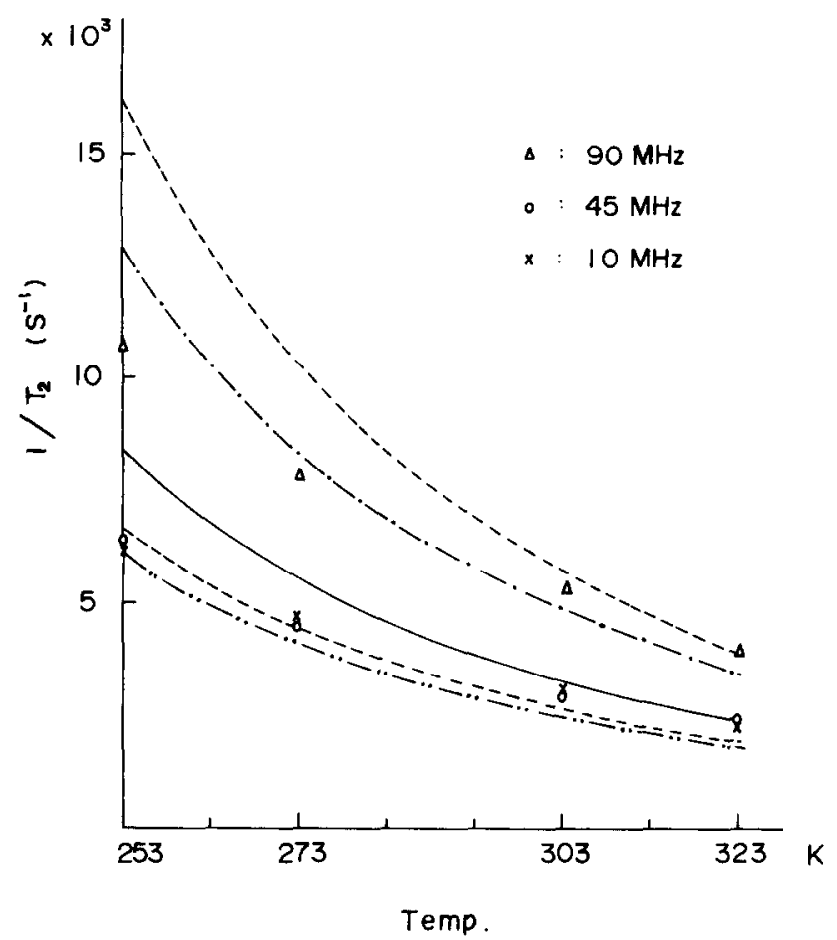

FIG. 3. Experimental and calculated proton spin-spin relaxation rates as a function of temperature. (a) (--) for model with $D=1.30 \times 10^{11} \mathrm{~s}^{-1}$ at $90 \mathrm{MHz}$, and (b) (-.-) for model with $D=1.30 \times 10^{11} \mathrm{~s}^{-1}$ at $45 \mathrm{MHz}$. The dashed curve above curve (a) (or (b)) is calculated at the same field as in (a) (or (b)) but with $D=1.12 \times 10^{11} \mathrm{~s}^{-1}$. The solid curve is for $10 \mathrm{MHz}$ and no distinction is found for the variation of $D$ between these two values.

(13). The incorporation of microviscosity into the Stokes-Einstein relation is to represent the picture of a structured liquid with clathrate-type vacancies, e.g.,

$$
\tau=\frac{4 \pi r^{3} \eta f}{3 k T}
$$

where $\eta$ is the solvent viscosity and the correction for microviscosity is

$$
f=\left(6 r_{s} / r+\left(1+r_{s} / r\right)^{-3}\right)^{-1} \text {. }
$$

The radius of the chloroform molecule, $r_{s}=2.95 \AA$, is employed, in accordance with the hexagonal closest packing model, for liquid chloroform. Also, by assuming that $\mathrm{Cr}(\mathrm{acac})_{3}$ is a sphere with a radius $r=6.20 \AA(2 b)$ one would then get a $32 \%$ reduction in viscosity $(f=0.32)$ in the use of chloroform as a solvent. In Fig. 4 , we have shown the results of overall reorientational correlation times as a function of $\eta / T$. The $\tau$ values deviate significantly from the Stokes-Einstein relation. The difference is more pronounced at low temperatures. These experimental results are clearly not explainable by simple hydrodynamic considerations and may well be due to interaction of the complex with the surrounding solvent molecules. This is consistent with the 


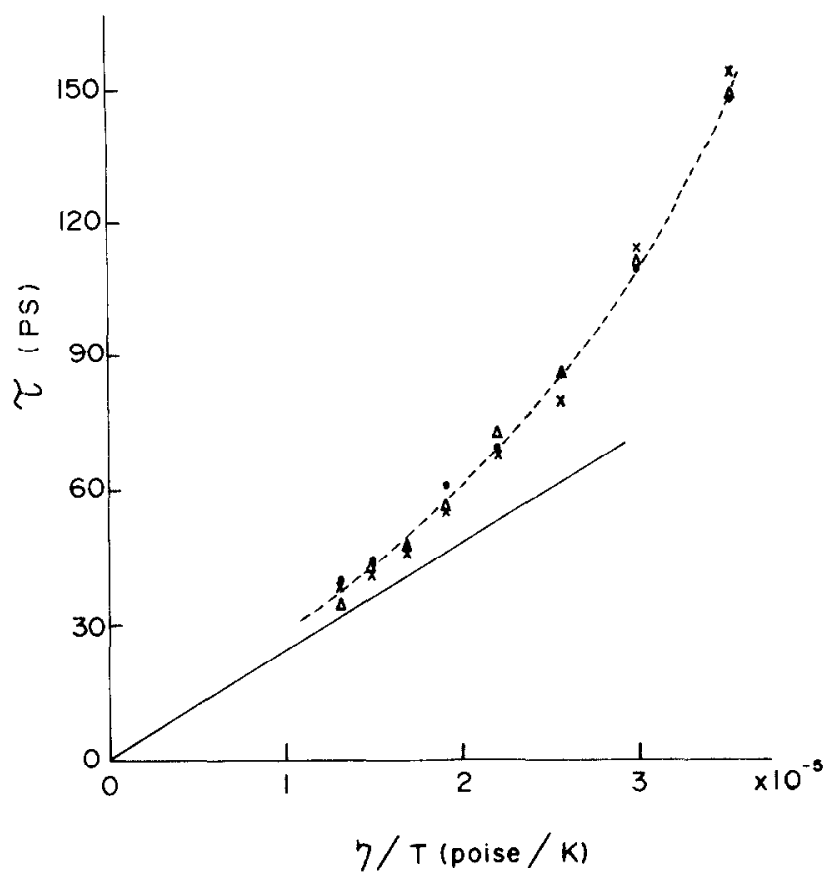

FIG. 4. Overall reorientational correlation times as a function of $\eta / T . O, \times, \Delta$, Denote values of $\tau_{\mathrm{CH}}$, $\tau_{\mathrm{CH}_{3}}$ and $\tau$, respectively, as shown in Table 1. The straight line is obtained in accordance with Eq. [20]. See the text for detailed information.

results of Frankel et al. (14), who observed that this complex has preferential solvation with chloroform in mixed solvents of $\mathrm{CHCl}_{3}$ and $\mathrm{CCl}_{4}$. The investigation was based on the effect of paramagnetic solutes on the linewidth of the solvent proton. Direct evidence may be found from the studies of temperature-dependent proton chemical shift of chloroform as shown in Fig. 5. In this system, a low molar ratio of chloroform is maintained in order to examine the temperature dependence of chemical shift. Because precipitation occurred, it was convenient only to study the region above 253 $\mathrm{K}$. The proton signal of chloroform in this solution is seen to have an upfield shift with respect to the proton resonance of pure chloroform. As the temperature is raised, the chemical shift varies toward the diamagnetic value. The analysis suggests possible solvation of the complex in chloroform solution.

\section{CONCLUSION}

In this work, proton relaxation rates have been measured for $\mathrm{CH}_{3}$ in dilute chloroform solution of $\mathrm{Cr}(\mathrm{acac})_{3}$. Frequency-dependent spin-lattice relaxation rates have been found below $35 \mathrm{MHz}$. The experimental frequency dependence of the spinlattice relaxation caused dominantly by dipolar coupling has been used to calculate reorientational correlation times. It is found that the effect of ESR relaxation on spinlattice relaxation is insignificant at magnetic fields above $35 \mathrm{MHz}$. The reorientational motion of this complex observed deviates significantly from that predicted for simple 


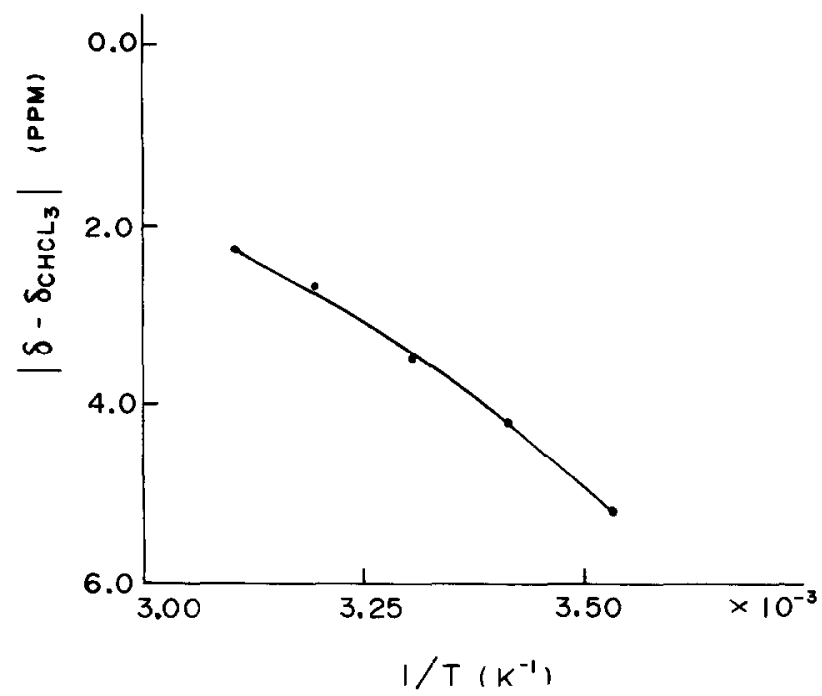

FIG. 5. Relative chemical shifts of chloroform, as referred to proton resonance of pure chloroform, versus $1 / T$ in $\mathrm{Cr}(\mathrm{acac})_{3}-\mathrm{CHCl}_{3}-\mathrm{CCl}_{4}$ system with their molar ratios 1:6:36.

Brownian motion. The deviation is believed to be attributable to the existence of various degrees of solvation of the complex at different temperatures. The solvation effects on ESR relaxation may be involved in the observed results.

Our calculation shows that scalar interaction is the dominant mechanism in spinspin relaxation at high field, while the dipolar interaction is the dominant one at low field. At $10 \mathrm{MHz}$, the scalar-dipolar cross relaxation contributes about $25 \%$ to the total spin-spin relaxation rate. The variation of ZFS improves the fitting with experiments only at high field. This fact rules out possible improvement for the observed rates at low field from the ESR effects of the present model.

\section{REFERENCES}

1. (a) B. R. MCGarvey, J. Chem. Phys. 40, 809 (1964); (b) M. Rubinstein, A. Baram, ANd Z. LuZ, Mol. Phys. 20, 67 (1971).

2. (a) G. C. LeVY AND J. D. CARGioli, J. Magn. Reson. 10, 231 (1973); (b) J. G. HeXeM, U. EdLund, AND G. C. LeVY, J. Chem. Phys. 64, 936 (1976).

3. D. M. Doddrell, D. T. PegG, AND M. R. Bendall, Chem. Phys. Lett. 40, 142 (1976).

4. L. P. Hwang and C. Y. Ju, J. Chem. Phys. 83, 3775 (1985).

5. T. MOELLER, Inorg. Synth. 5, 130 (1957).

6. A. ABRAGAM, "Principles of Nuclear Magnetism," Chap. 8, Oxford Univ. Press, Oxford, 1961.

7. L. S. Singer, J. Chem. Phys. 23, 379 (1955).

8. D. M. BRINK AND G. R. SATCHLER, “Angular Momentum," Oxford Univ. Press, Oxford, 1968.

9. B. Morosin, Acta Crystallogr. 19, 131 (1965).

10. J. B. LAMBERT, R. J. NiEHUIS, AND J. W. KeEPERS, Angew. Chem. Int. Ed. Eng. 20, 487 (1981).

11. (a) J. H. Freed, G. V. Bruno, AND C. PolnaszeK, J. Phys. Chem. 75, 3385 (1971); (b) J. H. Freed, in "Spin Labelling: Theory and Applications" (L. J. Berliner, Ed.), Chap. 3, Academic Press, New York, 1976.

12. D. R. J. BOYD AND H. W. ThOMPSON, Trans. Faraday Soc. 49, 128 (1953).

13. A. Gierer AND K. WiRTZ, Z. Naturforsch. A 8, 532 (1953).

14. L. S. Frankel, C. H. LANGFord, and T. R. Stengle, J. Phys. Chem. 74, 1377 (1970). 